\title{
Using a Mini-UAV to Support Wilderness Search and Rescue: Practices for Human-Robot Teaming
}

\author{
Michael A. Goodrich, Joseph L. Cooper, Julie A. Adams, Curtis Humphrey, Ron Zeeman, and Brian G. Buss
}

\begin{abstract}
Wilderness Search and Rescue can benefit from aerial imagery of the search area. Mini Unmanned Aerial Vehicles can potentially provide such imagery, provided that the autonomy, search algorithms, and operator control unit are designed to support coordinated human-robot search teams. Using results from formal analyses of the WiSAR problem domain, we summarize and discuss information flow requirements for WiSAR with an eye toward the efficient use of mUAVs to support search. We then identify and discuss three different operational paradigms for performing field searches, and identify influences that affect which human-robot team paradigm is best. Since the likely location of a missing person is key in determining the best paradigm given the circumstances, we report on preliminary efforts to model the behavior of missing persons in a given situation. Throughout the paper, we use information obtained from subject matter experts from Utah County Search and Rescue, and report experiences and "lessons learned" from a series of trials using human-robot teams to perform mock searches.
\end{abstract}

\section{INTRODUCTION}

Wilderness search and rescue (WiSAR) is the process of looking for a missing person in mountainous, desert, and other sparsely populated natural environments. In this paper, we identify a set of operational practices for using mini Unmanned Aerial Vehicles (mUAVs) to support WiSAR operations. We begin by introducing a fictionalized search scenario that helps identify key variables influencing the use of mUAVs in a WiSAR task. The scenario is motivated by a real situation, but significant facts have been altered for privacy reasons.

A call comes to the Sheriff's office reporting a missing person (MP). The MP's family reports that the person has been missing for approximately three hours. The family is concerned because the MP was in poor health. The family believed that the person was most likely to go to the Gate Cliffs area, shown in Figure 1. A Sheriff's department officer drives to a campground in the area and spots the MP's motorcycle in the bushes. At this point, the Sheriff's office calls out the Gate County Search and Rescue team.

Of the members of the search and rescue team, only a handful are trained to be incident commanders. One of these trained commanders volunteers for this search. She conducts a preliminary survey of area maps and makes the following observations:

- The Gate Cliffs area is a relatively narrow area of rugged but navigable terrain located on the side of William's Mountain.

- The terrain to the east, south, and southwest includes many cliffs and is extremely steep and rugged. There is only one dirt road through the area, and three or four

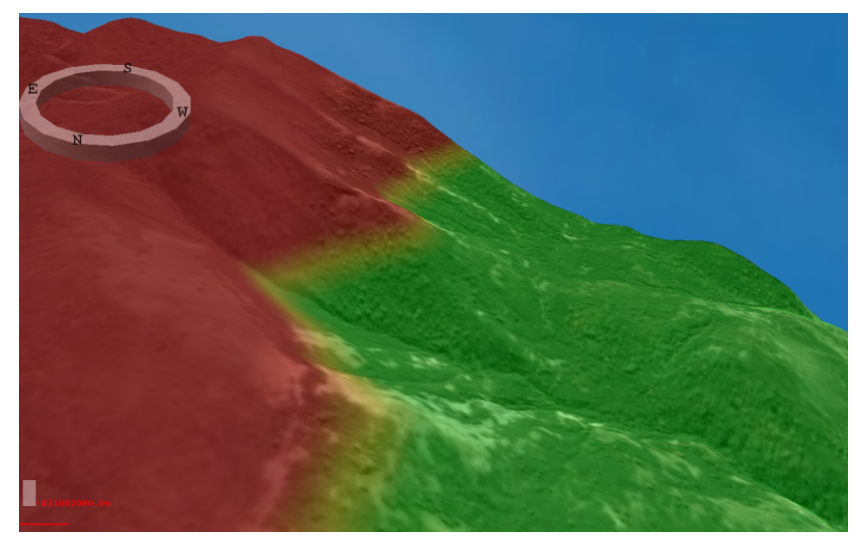

Fig. 1. View of the topology of the Gate Cliffs area facing south. To give a sense of scale, the plateau is approximately 1500 ' wide. The different shades indicate the altitude of the terrain relative to the mUAV.

hiking trails in the area, but two of these require technical rock climbing skills and equipment. Thus, mobility of ground searchers in the area will be very limited.

- The vegetation is significant enough to limit the ability of ground searchers to see around them, but sparse enough to allow views from the air; unfortunately, the steepness of the terrain limits the safe accessibility of the area for manned search aircraft.

- Using the motorcycle's location as the point last seen and assuming that the MP travels around $3 \mathrm{~km} / \mathrm{hr}$, the search area is already approximately $28 \mathrm{mi}^{2}$ and will grow between eight and ten square miles each hour for the next two hours.

Because of the rugged terrain and large search area, the incident commander wants to use the mini-UAV team to support the search effort.

The above scenario illustrates many of the factors that make mUAVs a potentially useful tool for performing WiSAR: a constrained by growing search area, rugged terrain, limitations in a ground searcher's field of view, and limitations on the use of manned aircraft. The purpose of this paper is to present efforts to support WiSAR search using mUAVs.

We assume a hierarchical organization typical of WiSAR efforts [16]. Leading the search effort is an incident commander who manages multiple search teams including several with technical search specialities such as rock-climbing and medical skills. The mUAV team is considered one such technical search team, and it includes a mission manager, a mUAV operator, a sensor operator, and ground searchers. Given this hierarchical 
organization, we emphasize how information requirements influence the coordination between humans and the mUAV. We begin with a brief survey of related work.

\section{RELATED Literature AND PREVIOUS WORK}

The experimental UAVs used in this work are small and light, with most having wingspans of approximately 42"-50" and flying weights of approximately 2 pounds. The airframes are derived from flying wing designs and are propelled by standard electric motors powered by lithium batteries. The autopilot is built on a small micro-processor, and is described in [1]. The standard sensor suite of the aircraft includes: 3-axis rate gyroscopes, 3-axis accelerometers, static and differential barometric pressure sensors, a GPS module, and a video camera on a gimballed mount. The test aircraft utilize 900 $\mathrm{MHz}$ radio transceivers for data communication and an analog $2.4 \mathrm{GHz}$ transmitter for video downlink. The mUAV uses the hierarchal control system described in [1].

Typically, UAVs engaged in a search task either require two operators or a single operator to fill two roles: a pilot, who "flies" the UAV, and a sensor operator, who interprets the imagery and other sensors. It is sometimes useful to include a third person to monitor the behavior of the pilot and sensor operator; this third person helps protect the pilot and sensor operator and helps provide greater situation awareness [2,6]. Complementing such search-specific work is general work on the human factors of UAVs $[3,5,12]$ and on the number of people required to manage an unmanned vehicle $[10,15]$.

The goal of this paper is to understand human factors issues related to fielded missions [2,4]. As a means of analyzing some of the human factors issues, we have conducted both a goal-directed task analysis [8] and a cognitive work analysis [19] of the WiSAR domain. These analyses are specific examples of more general work in human factors, aviation, situation awareness, etc. [7, 17].

In terms of search efficiency, literature related to manned aerial search is particularly relevant [14]. This work suggests that the goal of $100 \%$ target detection is in continual conflict with the goal of searching the largest area possible. This conflict means that resource allocation is a key problem in human-robot teams for the WiSAR domain.

\section{INFORMATION REQUIREMENTS IN WISAR}

In introducing human-robot teams into a problem domain that has previously been performed by human teams, it is important to understand the way the task is currently handled by humans. Since introducing mUAVs into the WiSAR domain should complement and support existing teams, it is especially important to understand the type of and timing of information in WiSAR. To encapsulate this, we have previously performed a goal-directed task analysis (GDTA) [8] and a partial cognitive work analysis (CWA) [19] of the WiSAR domain [9].

Rather than present the GDTA and CWA details in this paper, we can use this information to identify the central information flow of the process and use this process model to guide our analysis of human-robot WiSAR teams. As shown in Figure 2, the search task involves gathering evidence, utilizing that information to modify the understanding of the search problem, and then directing further efforts at additional evidence.

The information flow for WiSAR personnel begins with the initial details given by the reporting party. Responders immediately consider the urgency of the call based on the potential danger to the missing person and other factors. Combining prior knowledge and experience with information provided by the reporting party, responders develop a model of high probability sources of additional evidence. Potential sources of evidence are geographic locations surrounding the missing person's point last seen, but also include people familiar with the missing person or the missing person's bedroom or other property.

After evaluating initial sources of evidence, the WiSAR team develops and executes a plan for acquiring additional evidence. In the more challenging situations, the plan must allocate human and mUAV search resources to efficiently accumulate evidence from different sources. Such allocation is governed by the probability that useful information will be obtained, by the risks involved in gathering the information, and by the capabilities of available resources for acquiring information.

Time and additional evidence result in adjustments to the probability model of possible sources of evidence; changes in the model lead to changes to the search plan. All evidence changes the expected utility of searching in different areas. Incident command continually evaluates evidence and redirects available resources in order to maximize the value of the search.

Ideally, the search ends when the WiSAR team locates the missing person (probability distribution moves to a single spike). Work then proceeds on to rescue or recovery. However, the process may also end if the search continues long enough that the probability of the missing person actually being within the search area falls below a certain threshold or if dangers or other constraints (i.e., another incident) cause the relative expected value of continuing the search to fall below a threshold.

Given this information flow model, it is important to define how human robot teams can efficiently function to gather evidence given the capabilities and limitations of the team members. In the next section, we present observations from a series of field trials and simulation exercises on how humanrobot WiSAR teams should be organized.

\section{The mUAV TeChNicAl SEARCh TEAM}

The information flow model from the previous section suggests that search is the process of removing uncertainty about the location of the MP. This occurs either by finding signs that focus probabilities to particular locations or by reducing the probability in other regions by failing to find signs of the MP. The presence or absence of signs allows search resources to be shifted or refocused. To understand how human-robot teams can efficiently perform this search, we begin by analyzing 


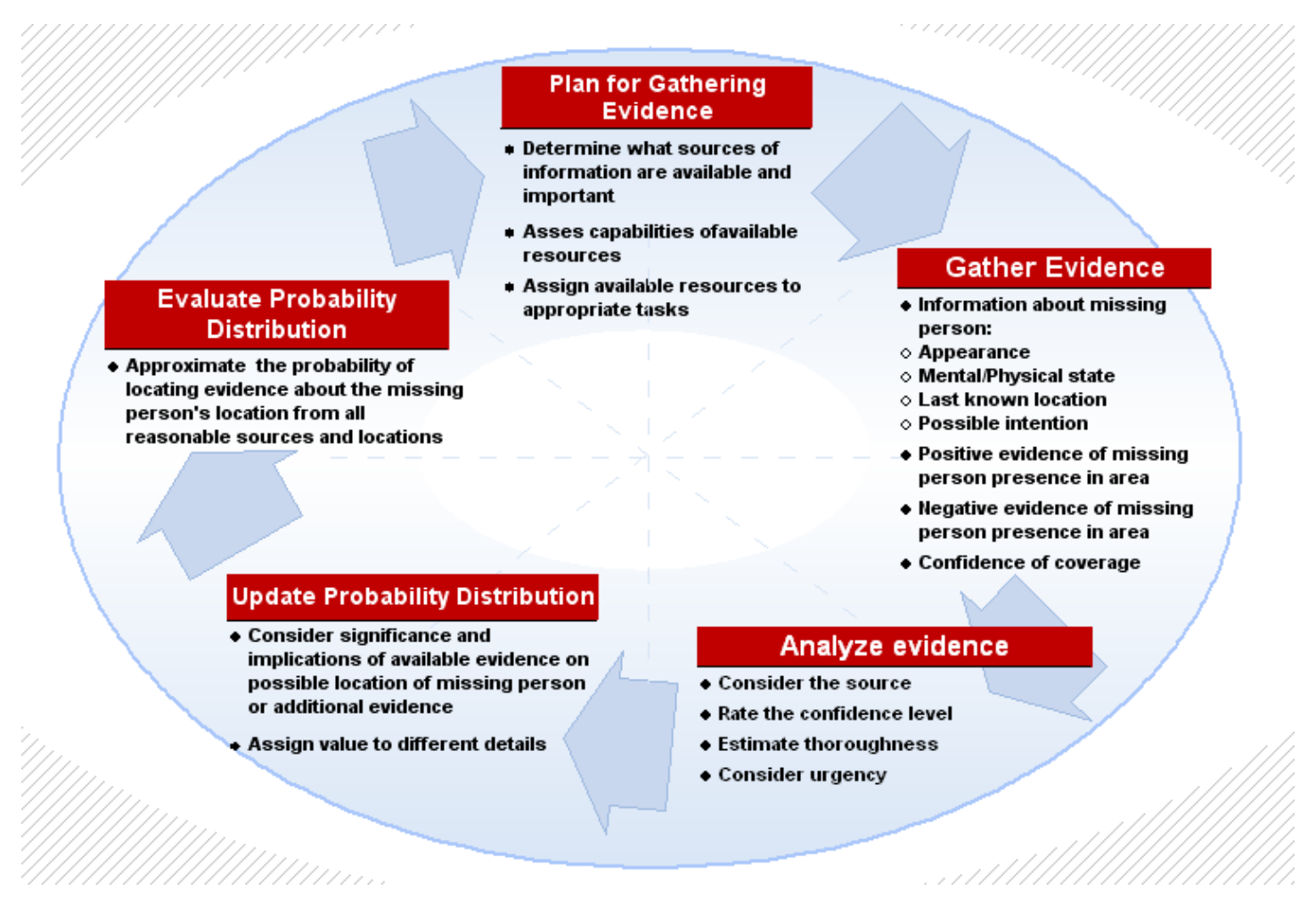

Fig. 2. Information flow in the WiSAR domain.

team roles. We then present three paradigms of coordinating these roles, describe scenarios where these paradigms were appropriate in a field trial, and summarize principles for selecting a paradigm given the circumstances in a search.

\section{A. mUAV-Supported WiSAR Roles}

WiSAR field tests, as well as similar tests from other search-related domains $[2,6]$, strongly suggests that multiple roles must be performed. In WiSAR, these roles include: UAV operator, sensor operator, mission manager, and ground support. These roles can theoretically be filled by one or more people with varying levels of authority, often supported by various autonomy algorithms and user interface technologies.

The UAV operator is responsible for guiding the UAV to a series of locations that allow the camera to obtain imagery of potential signs. The sensor operator is responsible for directing, for example, a gimballed camera and for inspecting/interpreting imagery to detect potential signs of the MP. The mission manager is responsible for managing the progression of the search with an emphasis on processing information, focusing search efforts, and re-prioritizing efforts. Ground support involves people in the field confirming or de-confirming signs by, for example, inspecting a brightly colored spot to see if it is a man-made object discarded by the MP. An operational assumption is that seeing a sign from the ground removes more uncertainty than seeing a sign from the air. An additional assumption is that the ground team can give feedback to the mUAV operator that improves situation awareness.

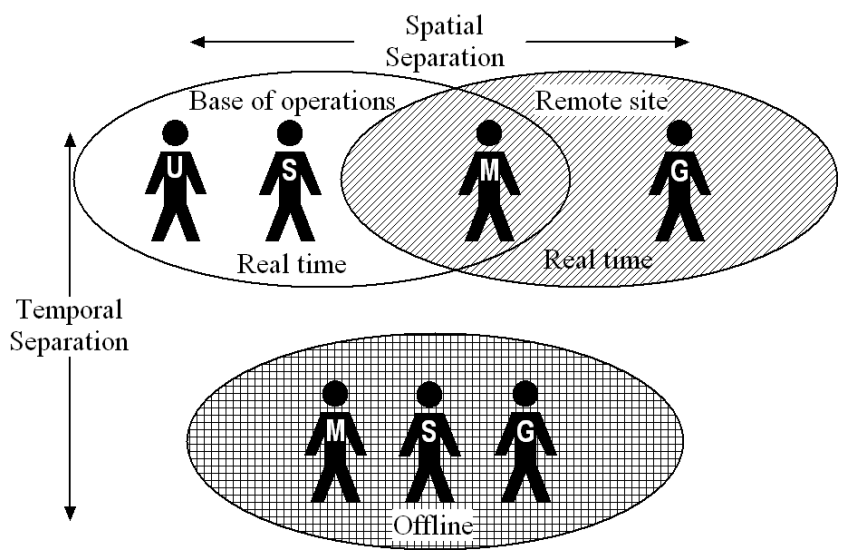

Fig. 3. Spatial and temporal relationships between people filling WiSAR roles: $\mathrm{U}=$ mUAV Operator, $\mathrm{S}=$ Sensor Operator, $\mathrm{M}=$ Mission Manager,and $\mathrm{G}=$ Ground Searchers.

The agents performing the roles can be located in three different physical locations: (1) aboard the aircraft (e.g., a program in the onboard computer), (2) at or near the location of the operator control unit (referred to as the base of operations), or (3) in the search area itself (referred to as the remote site). For this paper, we assume that basic aviation and navigation abilities such as attitude control or going to a waypoint are onboard the computer, and we omit discussion of such things as onboard target detection. Instead, we focus on how to organize people at the base of operations and the remote site. As illustrated in Figure 3, the important 
distinctions between the three operational paradigms discussed below are in the physical locations of the people filling the roles and the temporal sequence in which the roles are fulfilled.

\section{B. Sequential Operations}

Organization. In sequential operations, the mission manager works with the sensor and mUAV operators to create a search plan. The mUAV operator than executes this search plan and the resulting video and telemetry information is given to the mission manager and sensor operator. They evaluate the information with the goal of searching for signs of the MP. If a potentially valid sign is found, a ground support searcher is dispatched to the location to evaluate the sign. Information from ground searcher is then given back to the mission manager, and a new plan is created.

Scenario. A field trial ${ }^{1}$ similar to the scenario used in the introduction were performed. This scenario is a good example of when sequential operations can be used. In planning the field trial, it was obvious that typical (e.g., spiral and lawnmower) search patterns were inappropriate for the terrain because of the steepness in the east-west direction and because of a prevailing north wind. A more efficient search was to fly ellipses that tracked the mountain on one arch, curved away from the mountain and returned to near the starting point. During the return, the altitude and center of the ellipse was adjusted so that contours of the side of the mountain were searched. The major axis of the search ellipse fell along a north-south direction and the minor axis fell along an eastwest direction.

Principles. Sequential search is appropriate when there is limited ground mobility or when the probability of MP locations is large and uniformly distributed. Sequential search allows the team to gather data using the mUAV, cluster signs, and then dispatch a ground search team to the highest probability locations.

\section{Remote-Led Operations}

Organization. In remote-led operations, the mission manager joins the ground team to perform a ground-based, hasty search [9] such as tracking a footprint trail or using a canine team to track a scent trail. The mUAV operator flies an orbit that is centered on the location of the ground searchers while the sensor operator controls the camera to gather imagery beyond outside of what the ground searchers can see. Thus, the mUAV effectively extends what can potentially be seen by the ground searchers. This allows the mission manager greater access to potentially relevant information to guide the hasty search.

Scenario. In a field trial, we dispatched a ground team to track a mock trail of footprints. The trail lead through limited vegetation (sage brush and juniper trees) and over mildly hilly terrain (roughly plus or minus 100 meter deviation from the launch point altitude). As the ground searchers followed the

\footnotetext{
${ }^{1}$ There were some technical difficulties with the mUAV on this field trial, so many of the field observations are supplemented by observations from a simulator study and from thought experiments.
}

trail, the sensor operator looked for signs of the MP - signs that the ground team could not detect because of the brush and hills. Several false alarms were reported by the sensor operator (and inspected and de-confirmed by the ground team). When the ground team lost the trail of the MP, the mUAV operator flew orbits that spiraled out from the last known position of the MP. Doing this, the mUAV was able to locate the MP on a hillside about 100 meters away from the ground searchers but well beyond their field of view.

Principles. Remote-led operations are appropriate when the mission manager has more awareness of search-relevant information at some location in the field than at the base station. This occurs when there is a cluster of signs that allow the mission manager to rapidly update the model of the MP's location, such as might occur when tracking the individual. The mUAV provides supplementary information that broadens the scope of what the mission manager can include in his or her model.

\section{Base-Led Operations}

Organization. In base-led operations, the mission manager is located with the mUAV and sensor operators near the operator control unit. As the sensor operator identifies possible signs in the video, the mission manager adjusts his or her model of the likely locations of the MP and instructs the mUAV operator to focus flight time in high probability areas. Ground searchers follow the center of the mUAV track so that they are within a minimum distance from possible signs detected by the sensor operator. When the mUAV records a possible sign, the ground searchers can rapidly confirm or deconfirm the sign.

Scenario. In a field trial, we used this paradigm to perform a search. The mission manager translated the scenario description into a likely path taken by the MP. The mUAV operator translated this likely path into a set of waypoints that were placed in a queue. The mUAV then sequentially orbited the waypoints while the ground searchers followed the waypoints. When the sensor operator identified a sign, the ground team immediately investigated. After several false signs were deconfirmed by ground searchers, the sensor operator detected a true sign that was rapidly confirmed by the ground team to be the MP. The telemetry of the mUAV in this field test is shown in Figure 4.

Principles. Base-led operations are appropriate when the terrain allows for ground teams to be highly mobile but when there is not enough information to perform a hasty search. The ground team can position themselves at the center of a moving search orbit so that they are within minimal expected distance when the sensor operator detects a sign. Feedback from the ground allows the mission manager to adapt the search plan rapidly.

\section{Using Models of Missing Person Location}

In the previous section, we identified elements of search scenarios that influenced the best way to use a mUAV in WiSAR. Each of these scenarios relied heavily on information 


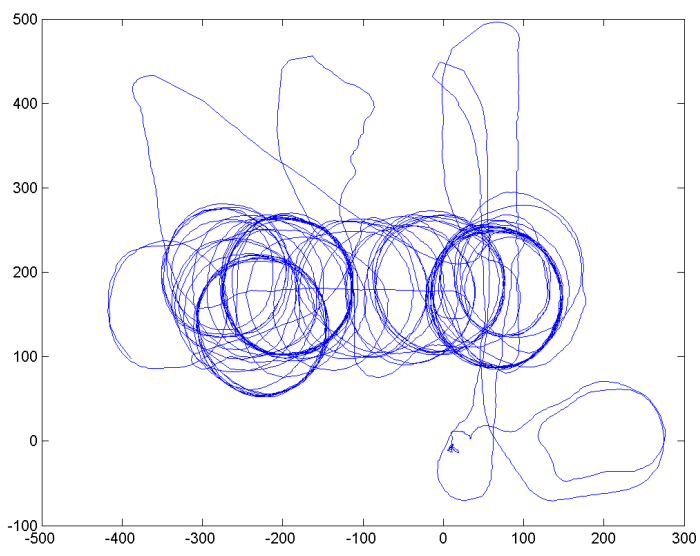

Fig. 4. Telemetry from a base-led field test. The mUAV begins at the launch point $(0,0)$, and then performs orbits a series of waypoints as the mission manager directs the search. The axes are meters to the north and east from launch point.

about the likely location of the MP such as the direction of travel of recent signs that the MP was in an area.

In any lost-person incident, efficient allocation of resources requires some prior assumption concerning the areas likely to contain the victim. Estimating the probability of area for a specific individual then becomes a significant priority at each stage of the search management. The probability of area (POA) assigned to any region indicates the belief that the lost person may be located within that region. As new information is gathered, the beliefs are updated; revised POA values are used to direct the continuing search effort until the mission is completed or suspended. Several methods are commonly used to generate initial probability distributions, including the local case histories, statistical prediction, and the Matson consensus method [16]. One approach which may provide a more specific probability distribution for a given incident involves simulation. Given a stochastic model of lostperson behavior and a geographic description of a particular region, a large number of simulations may be run. If the model is well matched to a particular victim, the distribution of the simulation agents at any time can be said to approximate the actual probability distribution of the victim's location. The resulting distribution may then be incorporated into the search planning and management resources.

\section{A. A Formalization of the Model.}

The basis of a general model may be constructed by letting the particle velocity be dynamically influenced by the environmental and motivational factors. We lump these factors into an abstract state variable that includes, for example, the local gradient. This model assumes that people tend to travel in approximately the same direction, with small perturbations influencing this direction of travel. This assumption reflects a tendency for people to travel in what they believe is a straight line, though this line tends to not be straight because of influences of the environment and the psychology of being lost [13]. Thus, the model is approximately a random walk, but the randomness affects velocity rather than position.

In the model, the perturbations should not be uniform; rather, they should be influenced by state or the characteristics of the environment and attributes of the missing person. For example, the most simple model presents perturbations biased slightly in a downhill direction. Extensions of this model provide directions influenced by vegetation density, trails, experience, motivation, etc.

This random walk model on velocity must be bounded by physical and natural constraints. The first constraint is that the magnitude of the velocity cannot exceed the maximum speed of a human on foot. Thus, the random walk on velocity includes reflecting boundaries to keep the velocity below a maximum. The second constraint is that people cannot traverse slopes that exceed a certain threshold because they are too steep to climb or too sleep to safely descend ${ }^{2}$. Thus, the random walk on velocity includes reflecting points that make certain directions of travel very rare - those directions that exceed the slope threshold.

According to Hill [13], it is not uncommon for missing persons to switch between various "reorientation strategies." Switching between strategies often includes a sudden change of direction. It can also include stopping for a moment. These strategy switches can be triggered by environmental stimuli, such as coming across a trail or hearing a sound.

Mathematically, the following model can include the above factors:

$$
v_{t+1}=\alpha_{t}(s)\left(v_{t}+\rho_{t}(s)\right)+\left(1-\alpha_{t}(s)\right) \eta_{t}(s)
$$

In this equation, $v_{t}$ indicates the velocity (speed and direction) at time $t, \rho_{t}(s)$ is a perturbation on this velocity at time $t$ as a function of environmental state $s, \alpha_{t}(s) \in\{0,1\}$ is a random and state-dependent factor that represents a relatively rare occurrence of suddenly switching from the current direction, and $\eta_{t}(s)$ is a state dependent and random new velocity that results when the missing person changes direction. Note that most of the time $\alpha_{t}(s)$ is unity, meaning that under nominal conditions a random walk in velocity occurs with reflecting boundaries $\left\|v_{t}\right\|=\min \left(\left\|v_{t}\right\|,\left\|v^{\max }\right\|\right)$ where $\left\|v^{\max }\right\|$ is the maximum velocity allowed.

By making the random variables state dependent, we can include not only temporal random variations, but also environmental triggers. For example, a nominal model of a missing person would have $\rho_{t}(s)$ distributed according to a normal distribution with small variance and a mean pointing in the direction of steepest slope. More generally, the behavior of the MP is strongly influenced by the experience and personality of the MP. Thus, it makes sense to allow the random variables to be influenced by these personality attributes.

\footnotetext{
${ }^{2}$ Note that missing persons can fall down such slopes, so this possibility needs to be considered in the model.
} 


\section{B. Toward Evaluating the Model.}

In order to verify that a particular model is a good predictor of actual victim behavior, the results of a collection of varied simulations must be compared with the observed behavior of actual victims. That is, we want to show that the positions of a sample of simulation agents and that of a sample of actual victims are distributed similarly. Our evaluation will be based upon the goodness-of-fit of the simulated and observed distributions. Two sources of observed distributions will be used in the evaluation: distributions of locations where MPs were found [18], and distributions generated by subject matter experts for a particular situation.

We have performed a preliminary analysis comparing simulation models to observed distribution. The goal of this analysis is to identify which parameters are most important in the model. We use the rms value of the K-S statistic during the simulation. This value facilitates comparison to determine which model results in a distribution which is, loosely, closest to the observed distribution for the longest time. This is somewhat artificial since the distribution of actual MP locations in [18] does not reveal any time history; the various victims were found after different intervals from the time they became lost.

Of the parameters in Equation (1), it seems that the probability of random changes and the maximum speed have the greatest effect on model integrity over the ranges tested. That is, it seems that the best results are obtained when the probability of random change is very low and where maximum speed is appropriately constrained.

\section{Using the Model}

The model can be used to select which operational paradigm is appropriate, and to plan the mUAV's or ground searcher's path. Many algorithms exist for mUAV path planning, but fewer algorithms exist for performing prioritized search in an efficient manner [11]. Future work should include pathplanning to do prioritized coverage searches.

\section{CONCLUSIONS}

A team of humans and mUAVs can be used to support WiSAR. Such a team can fit into the existing WiSAR information flow model, but the organization of the human-robot team roles depends strongly on situational factors, such as the density of MP signs, the mobility of ground searchers, and the ability of the mission manager to use information from the mUAV to modify the probability model. Simulations of likely MP behavior can help the mission manager form a model of the likely locations of a MP, but the mission manager should use simulation results as another source of information that affects team decisions. Field and simulation trials indicate that, based on the mission manager's model, sequential search, remote-led, or base-led operations can appropriately support WiSAR efforts.

\section{ACKNOWLEDGEMENTS}

The authors would like to thank the BYU MAGICC lab for their support. The work was partially supported by the National Science Foundation under grant number 0534736, by the Utah Small Unmanned Air Vehicle Center of Excellence, and by a Mentored Environment Grant from Brigham Young University.

\section{REFERENCES}

[1] R. Beard, D. Kingston, M. Quigley, D. Snyder, R. Christiansen, W. Johnson, T. McLain, and M. A. Goodrich. Autonomous vehicle technologies for small fixed-wing UAVs. Journal of Aerospace Computing, Information, and Communication, 2, 2005.

[2] J. L. Burke and R. R. Murphy. Human-robot interaction in USAR technical search: Two heads are better than one. In Proceedings of the 13th International Workshop on Robot and Human Interactive Communication (RO-MAN), Kurashiki, Okayama, Japan, 2004.

[3] G. L. Calhoun, M. H. Draper, M. F. Abernathy, M.Patzek, and F. Delgado. Synthetic vision system for improving unmanned aerial vehicle operator situation awareness. In J. G Verly, editor, Proceedings of SPIE Vol 5802, in Enhanced and Synthetic Vision 2005, 2003.

[4] J. Casper and R. R. Murphy. Human-robot interactions during the robotassisted urban search and rescue response at the world trade center. IEEE Transactions on Systems, Man and Cybernetics, Part B, 33(3):367-385, June 2003.

[5] J. L. Drury, J. Richer, N. Rackliffe, and M. A. Goodrich. Comparing situation awareness for two unmanned aerial vehicle human interface approaches. In Proceedings of the IEEE International Workshop on Safety, Security and Rescue Robotics (SSRR), Gaithersburg, Maryland, USA, August 2006.

[6] J. L. Drury, J. Scholtz, and H. A. Yanco. Awareness in human-robot interactions. In Proceedings of the IEEE International Conference on Systems, Man and Cybernetics, Washington D. C., October 2003.

[7] M. Endsley, B. Bolte, and D. Jones. Designing for Situation Awareness: An Approach to User-Centered Design. Taylor and Francis, London and New York, 2003.

[8] M. R. Endsley. A survey of situation awareness requirements in airto-air combat fighters. International Journal of Aviation Psychology, 3(2):157-168, 1993.

[9] M. A. Goodrich, M. Quigley, J. A. Adams, B. S. Morse, J. L. Cooper, D. Gerhardt, B. G. Buss, and C. Humphrey. Camera-equipped mini UAVs for wilderness search support: Tasks, autonomy, and interfaces. Technical Report BYUHCMI TR 2007-1, Brigham Young University, Provo, Utah, USA, 2007.

[10] P. A. Hancock, M. Mouloua, R. Gilson, J. Szalma, and T. Oron-Gilad. Is the UAV control ratio the right question? Ergonomics in Design, 2006.

[11] S. Hansen, T. McLain, and M. Goodrich. Probabilistic searching using a small unmanned aerial vehicle. In Proceedings of AIAA Infotech@Aerospace, California, May 7-10 2007.

[12] Manning the unmanned. In 2004 Human Factors of UAVs Workshop, Chandler, Arizona, May 2004. Cognitive Engineering Research Instutitue.

[13] K. A. Hill. The psychology of lost. In Lost Person Behavior. National SAR Secretariat, Ottawa, Canada, 1998.

[14] B. O. Koopman. Search and Screening: General Principles with Historical Applications. Pergamon Press, 1980. This book has been reprinted in its entirety in 1999 by the Military Operations Research Society, Inc.

[15] D. R. Olsen, Jr. and S. B. Wood. Fan-out: Measuring human control of multiple robots. In Proceedings of Human Factors in Computing systems, pages 231-238, 2004.

[16] T. J. Setnicka. Wilderness Search and Rescue. Appalachian Mountain Club, 1980.

[17] T. B. Sheridan. Humans and Automation: System Design and Research Issues. John Wiley and Sons, 2002.

[18] W. G. Syrotuck. An Introduction to Land Search: Probabilities and Calculations. Barkleigh Productions, Mechanicsburg, PA, 2000.

[19] K. Vicenti. Cognitive Work Analysis: Toward Safe, Productive and Healthy Computer-Based Work. Lawrence Erlbaum Associates, 1999. 